\title{
ON AN ELECTROMAGNETIC INVERSE PROBLEM FOR DISPERSIVE MEDIA
}

\author{
By \\ L. V. WOLFERSDORF \\ Bergakademie Freiberg, Freiberg, Germany
}

\begin{abstract}
Recently, R. S. Beezley and R. J. Krueger [1] (see also [2]) and I. Lerche [6] considered some electromagnetic inverse problems for dispersive media, where the constitutive relation between the displacement field and the electric field in a homogeneous, isotropic, dielectric, dispersive medium is determined from measurements on monochromatic electromagnetic plane waves within the medium. For this aim Beezley and Krueger used the reflection behavior of the plane waves in the time domain, whereas Lerche utilized the absorption behavior of the waves in the frequency domain stressing the fact that decrement measurements are relatively easy to perform compared to phase measurements. He reduced the corresponding inverse problem for the whole space to a nonlinear Riemann-Hilbert problem for a holomorphic function in the upper half-plane and (not quite exactly) to an equivalent nonlinear singular integral equation which he solved in a linear approximation.

In the present paper we refine the analysis of Lerche and show that the nonlinear Riemann-Hilbert problem for his inverse problem can be easily solved in closed analytical form. Moreover, we deal with analogous problems for a semi-infinite and a finite slab correspondingly to the problems treated in the mentioned work of Beezley and Krueger and develop a method for solving these inverse problems, too.
\end{abstract}

1. Introduction. In a rapidly varying electromagnetic field the constitutive relation between the displacement field $\mathbf{D}(\mathbf{x}, t)$ and the electric field $\mathbf{E}(\mathbf{x}, t)$ in a homogeneous, isotropic, dielectric, dispersive medium can be taken in the general linear form (cf. [4, pp. 306-310], [5, Sec. 77])

$$
\mathbf{D}(\mathbf{x}, t)=\varepsilon_{0}\left[\mathbf{E}(\mathbf{x}, t)+\int_{0}^{\infty} G(s) \mathbf{E}(\mathbf{x}, t-s) d s\right]
$$

in the time domain, where $\varepsilon_{0}$ is the constant permitivity of free space and $G(t)$ is the susceptibility kernel which is supposed to be a continuous and summable function on $[0, \infty)$ with the limiting value $G(\infty)=0$.

Applying the Fourier transform to (1) provides the relation

$$
\mathbf{D}(\mathbf{x}, \omega)=\hat{\varepsilon}(\omega) \mathbf{E}(\mathbf{x}, \omega)
$$

Received March 20, 1990. 
in the frequency domain with the complex permitivity

$$
\hat{\varepsilon}(\omega)=\varepsilon_{0} \cdot \varepsilon(\omega), \quad \varepsilon(\omega)=1+\int_{0}^{\infty} G(t) e^{i \omega t} d t .
$$

The function $\varepsilon(\omega)=\varepsilon_{1}(\omega)+i \varepsilon_{2}(\omega)$ has the following analytic properties (cf. [5, Sec. 82]):

(i) The function $\varepsilon(\omega)$ is holomorphic in the upper half-plane $H$ and continuous on its closure $\bar{H}=H+R$. It takes the value 1 at infinity and the value $\varepsilon^{0}>1$, the relative dielectric constant of the medium, at the origin.

(ii) There is $\varepsilon(\omega) \neq 0$ on $\bar{H}$. The function $\varepsilon(\omega)$ is real-valued only on the imaginary axis $I$, where it decreases monotonically from the value $\varepsilon^{0}$ at the origin to the value 1 at infinity.

(iii) On the real axis $R$ is the real part $\varepsilon_{1}(\omega)$ of $\varepsilon(\omega)$ is an even function and its imaginary part $\varepsilon_{2}(\omega)$ is an odd function so that $\varepsilon(-\omega)=\overline{\varepsilon(\omega)}$.

Furthermore, there holds

$$
\varepsilon_{2}(\omega)>0 \text { for real } \omega>0,
$$

which physically means that there occurs a dissipation of energy in the medium (cf. [5, Sec. 80]).

Property (i) implies the well-known Kramers-Kronig dispersion relations which relate the values of the real and imaginary parts $\varepsilon_{1}(\omega)$ and $\varepsilon_{2}(\omega)$ of $\varepsilon(\omega)$ on $R$.

If, further, the susceptibility kernel $G$ is additionally a Hölder continuous function, we have the inversion formula to $(3)$

$$
\begin{aligned}
G(t) & =(2 \pi)^{-1} \int_{-\infty}^{\infty}[\varepsilon(\omega)-1] e^{-i \omega t} d \omega \\
& =\pi^{-1} \int_{0}^{\infty}\left[\varepsilon_{1}(\omega)-1\right] \cos \omega t d \omega+\pi^{-1} \int_{0}^{\infty} \varepsilon_{2}(\omega) \sin \omega t d \omega
\end{aligned}
$$

as $t>0$ and $G(t)=0$ as $t<0$, where the first integral in the last line of Eq. (5) must be understood as Cauchy principal value in general (cf. [3, Secs. 3-4]).

In the sequel we construct functions $\varepsilon(\omega)$ with properties (i)-(iii), which additionally have Hölder continuous boundary values on $R$ and a prescribed asymptotic behavior at infinity, and define the corresponding function $G(t)$ by Eq. (5). If the function $\varepsilon(\omega)-1$ goes to zero with an order greater than one as $\omega$ tends to infinity or is a holomorphic function in a half-plane $\operatorname{Im} \omega>-\delta, \delta>0$ (as is often the case in applications), then the function $G(t)$ thus defined is indeed a continuous and summable function on $[0, x)$ with $G(x)=0$ as supposed above. In particular, we then have

$$
G(+0)=\pi^{-1} \int_{0}^{\infty}\left[\varepsilon_{1}(\omega)-1\right] d \omega .
$$

From Maxwell's equations there follows the equation

$$
\Delta \mathbf{E}(\mathbf{x}, t)-\mu_{0} \partial^{2} \mathbf{D} / \partial t^{2}=0
$$

with the constant magnetic permeability of free space $\mu_{0}$ and

$$
\Delta \mathbf{E}(\mathbf{x}, \omega)+\mu_{0} \omega^{2} \mathbf{D}(\mathbf{x}, \omega)=0
$$


in the frequency domain. Together with Eqs. (2), (3) Eq. (7) yields the wave equation

$$
\Delta \mathbf{E}(\mathbf{x}, \omega)+c^{-2} \omega^{2} \varepsilon(\omega) \mathbf{E}(\mathbf{x}, \omega)=0
$$

with the velocity of light $c=\left(\varepsilon_{0} \mu_{0}\right)^{-1 / 2}$ of free space in the frequency domain.

We now consider a plane monochromatic wave for a transverse component of the electric field travelling in the direction of the positive $x$-axis:

$$
E(x, t)=A(\omega) \exp i(k x-\omega t) .
$$

Inserting Eq. (9) into Eq. (6) with Eq. (1), one obtains the dispersion relation

$$
k^{2}=c^{-2} \omega^{2} \varepsilon(\omega)
$$

for the wave number $k(\omega)$. Writing, as usual,

$$
k=c^{-1} \omega(n+i \kappa)
$$

with $n$ the refraction index and $\kappa$ the absorption coefficient of the medium, it follows that

$$
\varepsilon(\omega)=\varepsilon_{1}(\omega)+i \varepsilon_{2}(\omega)=(n+i \kappa)^{2}
$$

and $\varepsilon_{1}=n^{2}-\kappa^{2}, \varepsilon_{2}=2 n \kappa$. On the real axis $R$ we have

$$
\begin{aligned}
& n(\omega)=\left(\left[\varepsilon_{1}+\left(\varepsilon_{1}^{2}+\varepsilon_{2}^{2}\right)^{1 / 2}\right] / 2\right)^{1 / 2}, \\
& \kappa(\omega)= \pm\left(\left[-\varepsilon_{1}+\left(\varepsilon_{1}^{2}+\varepsilon_{2}^{2}\right)^{1 / 2}\right] / 2\right)^{1 / 2} \quad \text { as } \omega \gtrless 0 .
\end{aligned}
$$

Like the function $\varepsilon(\omega)=f^{2}(\omega)$ the function $f(\omega)=n(\omega+i \kappa(\omega)$ has properties (i)-(iii) with the value $\left(\varepsilon^{0}\right)^{1 / 2}>1$ at the origin and the inequalities

$$
n(\omega)>0, \quad \kappa(\omega)>0 \text { for real } \omega>0 .
$$

There hold the Kramers-Kronig dispersion relations for $n$ and $\kappa$, in particular

$$
\begin{aligned}
n(\omega) & =1+\pi^{-1} \int_{-\infty}^{\infty} \kappa\left(\omega^{\prime}\right)\left(\omega^{\prime}-\omega^{-1} d \omega^{\prime}\right. \\
& =1+2 \pi^{-1} \int_{0}^{\infty} \omega^{\prime} \kappa\left(\omega^{\prime}\right)\left(\omega^{\prime 2}-\omega^{2}\right)^{-1} d \omega^{\prime} .
\end{aligned}
$$

2. Inverse problem. The simplest inverse problem consists in determining the function $G(t)$ from the given absorption coefficient $\kappa(\omega)>0$ for real $\omega>0$. This is equivalent to determining $G(t)$ from the ratio of the amplitudes of the wave for two abscissas $x_{1}, x_{2}$ since

$$
\left|E\left(x_{2}, t\right)\right| /\left|E\left(x_{1}, t\right)\right|=\exp \left(-\Delta c^{-1} \omega \kappa\right), \quad \Delta=x_{2}-x_{1} .
$$

In particular, we have $\delta=1 / \operatorname{Im} k=c \omega^{-1} \kappa^{-1}$ as the (logarithmic) penetration depth of the wave.

By means of Eq. (13) for $\kappa$ the problem is reduced to the nonlinear (quadratic) Riemann-Hilbert problem for the holomorphic function $\varepsilon(\omega)$ in the upper half-plane $H$ with the boundary condition

$$
\varepsilon_{2}^{2}-4 \kappa^{2} \varepsilon_{1}=4 \kappa^{4} \text { on } R
$$


and the condition $\varepsilon(\infty)=1$ at infinity (cf. [6, Eq. (26)]).

In the sequel we assume that the given function $\kappa(\omega)$ for real $\omega>0$ is a Höldercontinuous positive function on $(0, \infty)$ satisfying $\kappa(0)=\kappa(\infty)=0$ and a condition of the form $\kappa(\omega) \leq A \omega^{-\alpha}, \alpha>0$, for large $\omega>0$ (cf. [7, Sec. 43]). Then Eq. (15) yields the function $n(\omega)$ and hence $f(\omega)=n+i \kappa$ on $(0, \infty)$. By Eq. (12) we find $\varepsilon(\omega)$ on $(0, \infty)$ and finally by Eq. (5) the kernel function $G(t)$. The function $f(\omega)$ extended by $f(-\omega)=\overline{f(\omega)}$ to negative real $\omega$ solves the Riemann-Hilbert problem (16) and this solution is the unique one with this property. Further, the function $n(\omega)$ defined by Eq. (15) satisfies the first inequality in Eq. (14) if and only if the condition

$$
\int_{0}^{\infty} \omega^{\prime} \kappa\left(\omega^{\prime}\right)\left(\omega^{2}-\omega^{2}\right)^{-1} d \omega^{\prime}>-\pi / 2
$$

is fulfilled.

A more realistic model assumes that the dispersive medium occupies the region $x>0$ only whereas free space occupies the region $x<0$ (cf. [1]). A right-moving electromagnetic plane wave in the region $x<0$ impinges on the medium at normal incidence producing a left-moving reflected wave as well as establishing a transient field within the medium. Then we have Eq. (1) for the corresponding components $E, D$ in $x>0$ only whereas $D=\varepsilon_{0} E$ in $x<0$. This leads to the relation

$$
D(x, \omega)= \begin{cases}\varepsilon_{0} E(x, \omega) & \text { in } x<0 \\ \hat{\varepsilon}(\omega) E(x, \omega) & \text { in } x>0\end{cases}
$$

with the function $\hat{\varepsilon}$ given by Eq. (3) again in the frequency domain and to waves of the form

$$
E(x, t)= \begin{cases}A_{1}(\omega) \exp \left[i \omega\left(c^{-1} x-t\right)\right]+ & A_{2}(\omega) \exp \left[-i \omega\left(c^{-1} x+t\right)\right] \\ & \text { in } x<0, \\ B(\omega) \exp [i(k x-\omega t)] & \text { in } x>0 .\end{cases}
$$

The dispertion relation (10) holds true again. The relations of the coefficients $A_{1}$, $A_{2}, B$ to each other are determined by the continuity condition for $E$ and $E_{x}$ at $x=0$. But from Eq. (10) alone we obtain the same solution of the inverse problem as before, where, of course, now the amplitudes of the electric field are measured at two abscissas $x_{1}, x_{2}>0$.

Finally, we suggest the following approximating procedure for evaluating Eq. (15) in case the values $\kappa\left(\omega_{j}\right)>0$ of $\kappa$ are given at only finitely many frequencies $\omega_{j}>0$, $j=1, \ldots, N$. One uses the approximating ansatz

$$
\kappa(\omega) \approx \sum_{m=1}^{N} A_{m} \omega\left(\omega^{2}+a_{m}^{2}\right)^{-1}
$$

with given $a_{m}>0, m=1, \ldots, N$, and coefficients $A_{m}, m=1, \ldots, N$, satisfying the linear equation system

$$
\sum_{m=1}^{N} A_{m} \omega_{j}\left(\omega_{j}^{2}+a_{m}^{2}\right)^{-1}=\kappa\left(\omega_{j}\right), \quad j=1, \ldots, N,
$$


and obtains the approximating function

$$
n(\omega) \approx 1+\sum_{m=1}^{N} A_{m} a_{m}\left(\omega^{2}+a_{m}^{2}\right)^{-1} .
$$

The free choice of the $a_{m}$ can be further used to facilitate the numerical solvability of the system (21) and to fulfill the condition $n(\omega)>0$.

3. Further inverse problems. As in [1] we consider the case of a dispersive medium occupying a finite slab $0<x<L$, too. Then, Eq. (1) holds in $0<x<L$, whereas $D=\varepsilon_{0} E$ in $x<0$ and $x>L$, and correspondingly in Eq. (18) the first line holds in $x<0$ and $x>L$ and the second line holds in $0<x<L$. The electric field component is given by

$$
E(x, t)=\left\{\begin{array}{l}
A_{1}(\omega) \exp \left[i \omega\left(c^{-1} x-t\right)\right] \\
\quad+A_{2}(\omega) \exp \left[-i \omega\left(c^{-1} x+t\right)\right] \quad \text { in } x<0, \\
B_{1}(\omega) \exp [i(k x-\omega t)] \\
\quad+B_{2}(\omega) \exp [-i(k x+\omega t)] \text { in } 0<x<L, \\
C(\omega) \exp \left[i \omega\left(c^{-1} x-t\right)\right] \text { in } x>L,
\end{array}\right.
$$

where the dispersion relation (10) still holds true. The relations of the coefficients $A_{1}, A_{2}, B_{1}, B_{2}, C$ to each other are determined by the continuity condition for $E$ and $E_{x}$ at $x=0$ and $x=L$. In particular, we have

$$
B_{2}(\omega) / B_{1}(\omega)=\left(k-c^{-1} \omega\right)\left(k+c^{-1} \omega\right)^{-1} \exp (2 i k L) .
$$

In the first inverse problem the ratio of the amplitudes of the reflected and the transient wave within the medium is prescribed at an abscissa $x_{1} \in[0, L]$. Then the function $f(\omega)=n+i \kappa$ is to be determined from the expression

$$
\begin{aligned}
g(\omega) & =\left|k-c^{-1} \omega\right|\left|k+c^{-1} \omega\right|^{-1}\left|\exp \left[2 i k\left(L-x_{1}\right)\right]\right| \\
& =|f-1||f+1|^{-1} \exp \left(-A_{0} \omega \kappa\right), \quad A_{0}=2 c^{-1}\left(L-x_{1}\right),
\end{aligned}
$$

given for real $\omega>0$. By the inequalities (14) the function $g$ has to satisfy the inequality $0<g(\omega)<1$, and it is assumed as Hölder continuous. (If the ratio of the amplitudes of the transient wave is known at two abscissas $x_{1}, x_{2} \in[0, L]$ we again know the function $\kappa$ and have the inverse problem from above.)

In the following we extend $g(\omega)$ to negative real $\omega$ as an even function and assume that it has an asymptotic behavior at infinity of the form

$$
g(\omega) \sim g_{\infty} \omega^{-p} \text { as } \omega \rightarrow+\infty
$$

with $g_{\infty}>0$ and $p$ a positive integer. Correspondingly we look for holomorphic functions $f(\omega)$ in the upper half-plane $H$ with an asymptotic behavior at infinity of the form

$$
f(\omega) \sim 1+b_{p} \omega^{-p}
$$

wher $b_{p}=\delta_{p} a_{p}$ with $\delta_{p}=i$ if $p$ is odd, $\delta_{p}=-1$ if $p$ is even, and with $a_{p}>0$. For $p=2,3, \ldots$ the coefficient $a_{p}=2 g_{\infty}$ is uniquely determined by $g_{\infty}$. For 
$p=1$ this is also the case if $x_{1}=L$, whereas if $0 \leq x_{1}<L$ the asymptotic relation (27) can only hold for values $g_{\infty}$ satisfying the inequaltiy

$$
g_{\infty} \leq g_{m}=c\left[4 e\left(L-x_{1}\right)\right]^{-1}=\left(2 e A_{0}\right)^{-1}
$$

and $a_{1}$ is then determined by the equation

$$
a_{1} \exp \left(-A_{0} a_{1}\right)=2 g_{\infty},
$$

which has two roots for $g_{\infty}<g_{m}$ and one root for $g_{\infty}=g_{m}$.

At first we show the uniqueness of the solution $f$ to Eq. (25) with the prescribed asymptotic behavior (27). If $f_{1}$ and $f_{2}$ are two solutions of Eq. (25), we have $\mid \varphi_{1}\left(\omega|=| \varphi_{2}(\omega) \mid\right.$ on $R$ for the functions

$$
\varphi_{1,2}(\omega)=\left[f_{1}(\omega) \mp 1\right]\left[f_{2}(\omega) \mp 1\right]^{-1} \exp \left(A_{0} i \omega\left[f_{1,2}(\omega)-1\right]\right) .
$$

These are holomorphic functions in $H$, continuous on $\bar{H}$ since they are different from zero there, and satisfy $\varphi_{j}(\infty)=1$ for $p=2,3, \ldots, \varphi_{j}(\infty)=\exp \left(-A_{0} a_{1}\right)$ for $p=1$, where $j=1,2$. By the maximum and minimum property of the functions $\left|\varphi_{j}(\omega)\right|$ on $\bar{H}$ (or the uniqueness of the Dirichlet problem for the harmonic functions $\left.\ln \left|\varphi_{j}(\omega)\right|\right)$ then follows $\varphi_{1}(\omega)=\varphi_{2}(\omega)$ there, i.e.,

$$
C(\omega)\left[1-\exp \left(A_{0} i \omega \psi(\omega)\right)\right]=\psi(\omega)\left[1+\exp \left(A_{0} i \omega \psi(\omega)\right)\right]
$$

on $\bar{H}$, where $\psi(\omega)=f_{1}-f_{2}$ and $C(\omega)=f_{1} f_{2}-1$.

But the relation (30) can hold for small $|\omega|$ only if $\psi(\omega)=0$. For instance, for $\omega=i y, y>0$, we have the real equation

$$
B(y)\left[1-\exp \left(-A_{0} y \varphi(y)\right)\right]=\varphi(y)\left[1+\exp \left(-A_{0} y \varphi(y)\right)\right],
$$

where $B(y)=C(i y)$ and $\varphi(y)=\psi(i y)$, implying

$$
\varphi(y)=\left[1+\exp \left(-A_{0} y \varphi(y)\right)\right]^{-1} B(y) \exp \left(-\vartheta A_{0} y \varphi(y)\right) A_{0} y \varphi(y)
$$

with $0<\vartheta(y)<1$ so that $|\varphi(y)| \leq 2^{-1}|\varphi(y)|$ for sufficiently small $y>0$ and $\varphi(y)=0$. Therefore $\psi(\omega)=0$ and $f_{1}(\omega)=f_{2}(\omega)$ on $\bar{H}$.

For constructing the solution of the problem we introduce the function

$$
W(\omega)=(f(\omega)-1)(f(\omega)+1)^{-1} \exp \left[A_{0} i \omega(f(\omega)-1)\right]
$$

and write Eq. (25) in the form $|W(\omega)|=g(\omega)$ on $R$. Then the function

$$
W_{0}(\omega)=\delta_{p}^{-1} g_{\infty}^{-1}(\omega+i \varepsilon)^{p} W(\omega), \quad \varepsilon>0 \text { arbitrary },
$$

is holomorphic in $H$ and continuous and different from zero in $\bar{H}$ with $W_{0}(\infty)=1$ and satisfies the boundary condition

$$
\left|W_{0}(\omega)\right|=g_{0}(\omega), \quad g_{0}(\omega)=g_{\infty}^{-1}\left(\omega^{2}+\varepsilon^{2}\right)^{p / 2} g(\omega)
$$

on $R$, where $g_{0}(\infty)=1$. Therefore,

$$
W_{0}(\omega)=\exp w_{0}(\omega), \quad w_{0}(\omega)=(1 / \pi i) \int_{-\infty}^{\infty}\left(\omega^{\prime}-\omega\right)^{-1} \ln g_{0}\left(\omega^{\prime}\right) d \omega^{\prime}
$$


on $H$. Then the function $f(\omega)$ is the solution of Eq. (31), where

with

$$
W(\omega)=\delta_{p} g_{\infty}(\omega+i \varepsilon)^{-p} \exp w_{0}(\omega)
$$

$$
w_{0}(\omega)=\ln g_{0}(\omega)+(2 \omega / \pi i) \int_{0}^{\infty}\left(\omega^{\prime 2}-\omega^{2}\right)^{-1} \ln g_{0}\left(\omega^{\prime}\right) d \omega^{\prime}
$$

on $R$. I.e., one has to solve Eq. (31) for $f$ on $R$ with the given function

$$
\begin{aligned}
W(\omega)= & \delta_{p}\left(\omega^{2}+\varepsilon^{2}\right)^{p / 2}(\omega+i \varepsilon)^{-p} g(\omega) \\
& \cdot \exp \left(\frac{2 \omega}{\pi i} \int_{0}^{\infty}\left(\omega^{\prime 2}-\omega^{2}\right)^{-1} \ln \left[g_{\infty}^{-1}\left(\omega^{2}+\varepsilon^{2}\right)^{p / 2} g\left(\omega^{\prime}\right)\right] d \omega^{\prime}\right),
\end{aligned}
$$

where again $\delta_{p}=i$ for odd $p$, and $\delta_{p}=-1$ for even $p$. Like $f$ the function $W$ satisfies the relation $W(-\omega)=\overline{W(\omega)}$ on $R$ and Eq. (31) need be solved for real $\omega>0$ only. From the uniqueness of the solution $f$ with the prescribed asymptotic behavior (27) it follows that the function $W$ is also unique and hence independent of the chosen $\varepsilon>0$. Taking the limit $\varepsilon \downarrow 0$ we finally have

$$
W(\omega)=\delta_{p} g(\omega) \exp \left(\frac{2 \omega}{\pi i} \int_{0}^{\infty}\left(\omega^{\prime 2}-\omega^{2}\right)^{-1} \ln \left[g_{\infty}^{-1} \omega^{p} g\left(\omega^{\prime}\right)\right] d \omega^{\prime}\right)
$$

for real $\omega>0$. For the existence of a physically relevant solution of the inverse problem (25) it must be assumed that Eq. (31) has a solution $f$ satisfying the inequalities (14). We remark that by the uniqueness of $f$ the relation $f(-\omega)=\overline{f(\omega)}$ on $R$ for the solution of Eq. (31) is automatically fulfilled.

In the special case $x_{1}=L$ we simply have

$$
f(\omega)=[1+W(\omega)][1-W(\omega)]^{-1}
$$

and the inequalities (14) are satisfied if

$$
|W(\omega)|<1 \text { and } \operatorname{Im} W(\omega)>0
$$

for real $\omega>0$. The first condition of (35) (which is also sufficient for the regularity of the solution (34)) is fulfilled by $|W(\omega)|=g(\omega)$ and the assumption $0<g(\omega)<1$. By Eq. (33) the second condition of (35) leads to the existence condition

$$
-\pi^{2}<4 \omega \int_{0}^{\infty}\left(\omega^{\prime 2}-\omega^{2}\right)^{-1} \ln \left[g_{\infty}^{-1} \omega^{p} g\left(\omega^{\prime}\right)\right] d \omega^{\prime}<\pi^{2}
$$

for real $\omega>0$ if $p$ is odd, and

$$
0<2 \omega \int_{0}^{\infty}\left(\omega^{\prime 2}-\omega^{2}\right)^{-1} \ln \left[g_{\infty}^{-1} w^{\prime p} g\left(\omega^{\prime}\right)\right] d \omega^{\prime}<\pi^{2}
$$

for real $\omega>0$ if $p$ is even.

We conclude the consideration of the first inverse problem with the following two simple examples for the case $x_{1}=L$.

EXAMPLE 1 WITH $p=1$ :

$$
\begin{aligned}
g(\omega) & =\left(4 \omega^{2}+9\right)^{-1 / 2}, \quad a_{1}=1, \quad g_{0}(\omega)=\left(\omega^{2}+\varepsilon^{2}\right)^{1 / 2}\left(\omega^{2}+9 / 4\right)^{-1 / 2}, \\
W(\omega) & =(i / 2)(\omega+3 i / 2)^{-1}, \quad f(\omega)=1+i(\omega+i)^{-1}, \\
n(\omega) & =1+\left(1+\omega^{2}\right)^{-1}, \quad \kappa(\omega)=\omega\left(1+\omega^{2}\right)^{-1}, \quad G(t)=(2+t) e^{-t} .
\end{aligned}
$$


EXAMPLE 2 WITH $p=2$ :

$$
\begin{aligned}
g(\omega) & =\left(4 \omega^{4}+4 \omega^{2}+9\right)^{-1 / 2}, \quad a_{2}=1, \\
g_{0}(\omega) & =\left(\omega^{2}+\varepsilon^{2}\right)\left(\omega^{4}+\omega^{2}+9 / 4\right)^{-1 / 2}, \\
W(\omega) & =\left(3-2 \omega^{2}-4 \omega i\right)^{-1}, \quad f(\omega)=1-(\omega+i)^{-2}, \\
n(\omega) & =1+\left(1-\omega^{2}\right)\left(1+\omega^{2}\right)^{-2}, \quad \kappa(\omega)=2 \omega\left(1+\omega^{2}\right)^{-2}, \\
G(t) & =t\left(2+t^{2} / 6\right) e^{-t} .
\end{aligned}
$$

In the second inverse problem the ratio of the amplitudes of the sum of the reflected and transient wave within the medium is prescribed at two abscissas $x_{1}, x_{2}$ with $0 \leqq x_{1}<x_{2} \leqq L$. Then the function $f(\omega)=n+i \kappa$ is to be determined from the expression

$$
h(\omega)=h_{1}(\omega) \cdot h_{2}^{-1}(\omega)
$$

where $h_{j}(\omega)=\left|H_{j}(\omega)\right|, j=1,2$, with

$$
H_{j}(\omega)=(f+1) \exp \left[i c^{-1} \omega f x_{j}\right]+(f-1) \exp \left[i c^{-1} \omega f\left(2 L-x_{j}\right)\right]
$$

given for real $\omega>0$. There must hold $h_{j}(\omega)>0$ for real $\omega>0$ since $H_{j}(\omega)=0$ implies $g_{j}(\omega)=1$ for the function (25) with $A_{0}=2 c^{-1}\left(L-x_{j}\right)$ in contradiction to the inequalities (14). Therefore the given function $h$ is assumed to be Hölder continuous and positive with $h(0)=1$.

In the sequel we again extend $h(\omega)$ to negative real $\omega$ as an even function and assume further that it satisfies

$$
h(\omega) \sim h_{\infty} \text { as } \omega \rightarrow \infty,
$$

where $h_{\infty} \geqq 1$. Correspondingly we look for holomorphic functions $f(\omega)$ in the upper half-plane $H$ with an asymptotic behavior at infinity of the form

$$
f(\omega)=1+i a \omega^{-1}+o\left(\omega^{-1}\right), \quad a \geqq 0,
$$

implying $H_{j}(\omega) \sim 2 \exp \left[(-a+i \omega) c^{-1} x_{j}\right]$. Therefore the coefficient $a$ is uniquely determined by $h_{\infty}$ through the relation

$$
a c^{-1} \Delta=\ln h_{\infty}, \quad \Delta=x_{2}-x_{1} .
$$

For constructing a solution of the problem we introduce the function

$$
W(\omega)=H_{1}(\omega) \cdot H_{2}^{-1}(\omega)
$$

and write Eq. (38) in the form $|W(\omega)|=h(\omega)$ on $R$. Then the function

$$
\begin{aligned}
W_{0}(\omega) & =\exp \left[(-a+i \omega) c^{-1} \Delta\right] W(\omega) \\
& =h_{\infty}^{-1} \exp \left(i \omega c^{-1} \Delta\right) W(\omega)
\end{aligned}
$$

is holomorphic in $H$ and continuous and different from zero in $\bar{H}$ with $W_{0}(\omega)=1$, and satisfies the boundary condition

$$
\left|W_{0}(\omega)\right|=h_{0}(\omega), \quad h_{0}(\omega)=h_{\infty}^{-1} h(\omega)
$$


on $R$, where $h_{0}(\infty)=1$. Therefore,

$$
W_{0}(\omega)=\exp w_{0}(\omega), \quad w_{0}(\omega)=(1 / \pi i) \int_{-\infty}^{\infty}\left(\omega^{\prime}-\omega\right)^{-1} \ln h_{0}\left(\omega^{\prime}\right) d \omega^{\prime}
$$

on $H$. Then the function $f(\omega)$ is the solution of Eq. (43), where

$$
W(\omega)=h_{\infty} \exp \left(-i \omega c^{-1} \Delta\right) \exp w_{0}(\omega)
$$

with

$$
w_{0}(\omega)=\ln h_{0}(\omega)+(2 \omega / \pi i) \int_{0}^{\infty}\left(\omega^{2}-\omega^{2}\right)^{-1} \ln h_{0}\left(\omega^{\prime}\right) d \omega^{\prime}
$$

on $R$. I.e., one has to solve Eq. (43) for $f$ on $R$ with the given function

$$
\begin{aligned}
W(\omega)= & h(\omega) \exp \left(-i \omega c^{-1} \Delta\right) \\
& \cdot \exp \left(\frac{2 \omega}{\pi i} \int_{0}^{\infty}\left(\omega^{\prime 2}-\omega^{2}\right)^{-1} \ln \left[h_{\infty}^{-1} h\left(\omega^{\prime}\right)\right] d \omega^{\prime}\right) .
\end{aligned}
$$

Again it is sufficient to solve Eq. (43) for real $\omega>0$ only. For the existence of a physically relevant solution of the inverse problem (38) it must be assumed that Eq. (43) has a solution $f$ satisfying the inequalities (14).

Eq. (43) can also be written in the form

$$
K_{1}(\omega)=W(\omega) \cdot K_{2}(\omega),
$$

where

$$
\begin{aligned}
& K_{1}(\omega)=(f+1) \exp \left[-i c^{-1} \omega f \Delta\right]+(f-1) \exp \left[i c^{-1} \omega f\left(2 L-x_{1}-x_{2}\right)\right], \\
& K_{2}(\omega)=(f+1)+(f-1) \exp \left[i c^{-1} \omega f 2\left(L-x_{2}\right)\right] .
\end{aligned}
$$

In particular, in the simplest case $x_{1}=0, x_{2}=L$ we have the equation

$$
(f+1) \exp \left(-i c^{-1} \omega f L\right)+(f-1) \exp \left(i c^{-1} \omega f L\right)=2 W f .
$$

We finally show the uniqueness of the solution $f$ to Eq. (43) with the prescribed asymptotic behavior (41). In the case of Eq. (47) the difference $\psi=f_{1}-f_{2}$ of two solutions $f_{1}, f_{2}$ satisfies the equation

$$
C_{1}(\omega) \psi(\omega)+C_{2}(\omega)=2 W(\omega) \psi(\omega),
$$

where

$$
\begin{aligned}
C_{1}(\omega)= & \exp \left(-i c^{-1} \omega f_{1} L\right)+\exp \left(i c^{-1} \omega f_{1} L\right), \\
C_{2}(\omega)= & \left(f_{2}+1\right)\left[\exp \left(-i c^{-1} \omega f_{1} L\right)-\exp \left(-i c^{-1} \omega f_{2} L\right)\right] \\
& +\left(f_{2}-1\right)\left[\exp \left(i c^{-1} \omega f_{1} L\right)-\exp \left(i c^{-1} \omega f_{2} L\right)\right] .
\end{aligned}
$$

For $\omega=i y, y>0$, and the real-valued function $\varphi(y)=\psi(i y)$ we have the asymptotic relations

$$
C_{1}(\omega) \sim \exp \left[(y+a) c^{-1} L\right], \quad C_{2}(\omega) \sim 2 c^{-1} L y \varphi(y) \exp \left[(y+a) c^{-1} L\right]
$$

besides

$$
W(\omega) \sim \exp \left[(y+a) c^{-1} L\right] \text { as } y \rightarrow \infty
$$


so that due to (48) the relation

$$
\varphi(y) \sim 2 c^{-1} \operatorname{Ly\varphi }(y) \text { as } y \rightarrow \infty
$$

holds. Hence there follows $\varphi(y)=0$ for arbitrary large $y$ implying $\psi(\omega)=0$ on $\bar{H}$. Analogously, in the general case of Eq. (46) we obtain the asymptotic relation

$$
\varphi(y) \sim 2 c^{-1} \Delta y \varphi(y) \text { as } y \rightarrow \infty,
$$

which again yields $\psi(\omega)=0$ and $f_{1}(\omega)=f_{2}(\omega)$ on $\bar{H}$.

\section{REFERENCES}

[1] R. S. Beezley and R. J. Krueger, An electromagnetic inverse problem for dispersive media, J. Math. Phys. 26, 317-325 (1985)

[2] J. P. Corones, R. J. Krueger, and V. H. Weston, Some recent results in inverse scattering theory, Inverse Problems of Acoustic and Elastic Waves (ed. by F. Santosa, Y.-H. Pao, W. W. Symes, and Ch. Holland), SIAM, Philadelphia, 65-81, 1984

[3] R. B. Guenther and J. W. Lee, Partial Differential Equations of Mathematical Physics and Integral Equations, Prentice-Hall, Englewood Cliffs, N. J., 1988

[4] J. D. Jackson, Classical Electrodynamics, Wiley, New York, 1975

[5] L. D. Landau and E. M. Lifschitz, Lehrbuch der theoretischen Physik, Vol. VIII, Elektrodynamik der Kontinua, 4th ed., Akademie-Verlag, Berlin, 1985

[6] I. Lerche, Some singular, nonlinear integral equations arising in physical problems, Quart. Appl. Math. 44, 319-326 (1986)

[7] N. I. Muskhelishvili, Singular Integral Equations, Noordhoff, Groningen, 1953 